Europhysics Letters

PREPRINT

\title{
Re-entrant pinning of Wigner molecules in a magnetic field due to a Coulomb impurity
}

\author{
B. Szafran ${ }^{1,2}$ and F.M. Peeters ${ }^{1}$ \\ 1 Departement Natuurkunde, Universiteit Antwerpen (Campus Drie Eiken), B-2610 \\ Antwerpen, Belgium \\ 2 Faculty of Physics and Nuclear Techniques, AGH University of Science and Technol- \\ ogy, al. Mickiewicza 30, 30-059 Kraków, Poland
}

PACS. 73.21.La - Quantum dots.

PACS. 73.20.Qt - Electron solids.

\begin{abstract}
Pinning of magnetic-field induced Wigner molecules (WMs) confined in parabolic two-dimensional quantum dots by a charged defect is studied by an exact diagonalization approach. We found a re-entrant pinning of the WMs as function of the magnetic field, a magnetic field induced re-orientation of the WMs and a qualitatively different pinning behaviour in the presence of a positive and negative Coulomb impurity.
\end{abstract}

Low-density electron systems in bulk may form an ordered crystalline phase called Wigner crystal [1] in which electron charges are spatially separated. A similar collective type of electron localization in quantum dots (QDs) is called Wigner molecule (WM) [2]. WMs may be formed in large QDs [2] or be induced by a strong magnetic field [3] in the quantum Hall regime. Wigner localization is observed in the inner coordinates of the quantum system whose charge density conserves the symmetry of the external potential [4]. Therefore, in circular QDs $[4,5]$ the charge density will be circular symmetric even in the Wigner phase. However, a perturbation of the potential may pin [6] the charge density at a fixed orientation in the laboratory frame which should allow for the experimental observation [7] of Wigner localization. Pinning of the magnetic-field induced WMs by the anisotropy of the potential [8] or by an attractive Gaussian impurity potential [9] in the absence of a magnetic field have been studied previously. Here, we will show that the WM pinning is qualitatively very different in the presence of a positive and negative impurity.

We consider WMs induced by a magnetic field in a two-dimensional harmonic QD. A strong magnetic field polarizes the spins of the confined electrons and leads to the formation of a so-called maximum density droplet (MDD) corresponding to the lowest Landau level filling factor $\nu=1$. Stronger fields induce the MDD to decay into a molecular phase with $\nu<1$, for which the distribution of electrons in the inner coordinates resembles the equilibrium configuration of a classical point-charge system [10]. The external magnetic field increases the absolute value of the angular momentum of the confined electron system inducing its changes between certain 'magic' [11] values for which the classical distribution of electrons in the inner ('rotating') frame of reference can be realized.

(c) EDP Sciences 
In this letter we investigate the way in which the potential of a charged defect (donor or acceptor ion) situated outside the QD symmetry axis stops the "rotation" of the electron system and freezes the WM at a fixed orientation. We use the configuration interaction approach which allows for an exact solution of the few-electron Schrödinger equation. We found that at magnetic fields inducing the angular momentum transitions the exact groundstate can correspond to broken-symmetry charge density with semi-classical localization in the laboratory frame. Broken-symmetry charge distributions were previously obtained as artifacts of mean-field methods [4]. The existance of exact broken-symmetry states makes the WMs susceptible to pinning by an arbitrarily distant charge defect (donor or acceptor ion) at the angular momentum transitions. Consequently, a distant defect induces re-entrant WM pinning as function of the strength of the magnetic field. We show that the orientation of the pinned WMs can change with the magnetic field and demonstrate an essentially different pinning behavior for a positive and negative impurity.

We assume that the system of $N$-electrons is spin-polarized by the external magnetic field and that the electrons are confined to move in the $z=0$ plane. The present configuration interaction approach is constructed in the following way. The single-electron Hamiltonian for the considered system reads

$$
h=(-i \hbar \nabla+e \mathbf{A})^{2} / 2 m^{*}+m^{*} \omega^{2}\left(x^{2}+y^{2}\right) / 2+B s_{z} g^{*} \mu_{B} \pm e^{2} / 4 \pi \epsilon \epsilon_{0} r_{e d},
$$

where $m^{*}$ is the electron band effective mass, $\hbar \omega$ is the confinement potential energy, $\epsilon_{0}$ is the static dielectric constant, $(0,0, B)$ is the magnetic field vector, $s_{z}$ is the $z$-component of the electron spin, $g^{*}$ is the effective Landé factor and $r_{e d}$ is the distance between the electron and the charged defect. The sign in the last term of eq. (1) is $-(+)$ for a positively (negatively) charged defect. We apply the Landau gauge $\mathbf{A}=(-B y, 0,0)$ and adopt GaAs material parameters $m^{*}=0.067 m_{0}, \epsilon=12.9$ and $g^{*}=-0.44$ as well as $\hbar \omega=3 \mathrm{meV}$. Hamiltonian (1) is diagonalized in a multicenter basis $\Psi_{\mu}(\mathbf{r})=\sum_{i=1}^{M} c_{i}^{\mu} \psi_{\mathbf{R}_{i}}(\mathbf{r})$ with

$$
\psi_{\mathbf{R}}(\mathbf{r})=\sqrt{\alpha} \exp \left\{-\alpha(\mathbf{r}-\mathbf{R})^{2} / 4+i e B(x-X)(y+Y) / 2 \hbar\right\} / \sqrt{2 \pi},
$$

where $\mathbf{R}=(X, Y)$. The single electron wave functions $\Psi_{\mu}$ are subsequently used for construction of $M ! / N !(M-N)$ ! Slater determinants - the basis set for diagonalization of the $N$-electron Hamiltonian. $\alpha$ and the positions of the centers $\mathbf{R}_{i}$ are chosen such that they minimize the total energy. Function (2) with $\alpha=e B / \hbar$ is the lowest Landau level eigenfunction. The basis set of displaced functions (2) allows for a very precise determination of the exact Fock-Darwin [4] energy levels, including higher Fock-Darwin bands, which at strong magnetic fields tend to excited Landau levels. We have verified the accuracy of the present approach comparing its results with the standard exact diagonalization method [14]. We have taken 12 centers placed on a circle. Above the MDD decay $(B>5.8,4.85$ and $4.65 \mathrm{~T}$ for 2,3 and 4 electrons) and below $20 \mathrm{~T}$ the overestimation of the exact energy for 2,3 and 4 electrons is lower than $0.01,0.06$ and $0.12 \mathrm{meV}$ respectively. Few-electron wave functions calculated in the Landau gauge are not eigenfunctions of the angular momentum, but using the gauge-independent expectation value of its operator we can look at the angularmomentum transformations of the confined system. The precision in the determination of the critical fields inducing ground-state transformation is better than 0.15 T. Previously, displaced Landau level functions (2) were used in the investigation of the WMs with approximate approaches, i.e., single-determinant of non-orthogonal wave functions [15], Hartree-Fock [16], and rotated-electron-molecule approach [17]. Due to the arbitrariness in the choice of centers the present configuration interaction approach can be easily applied to potentials without 

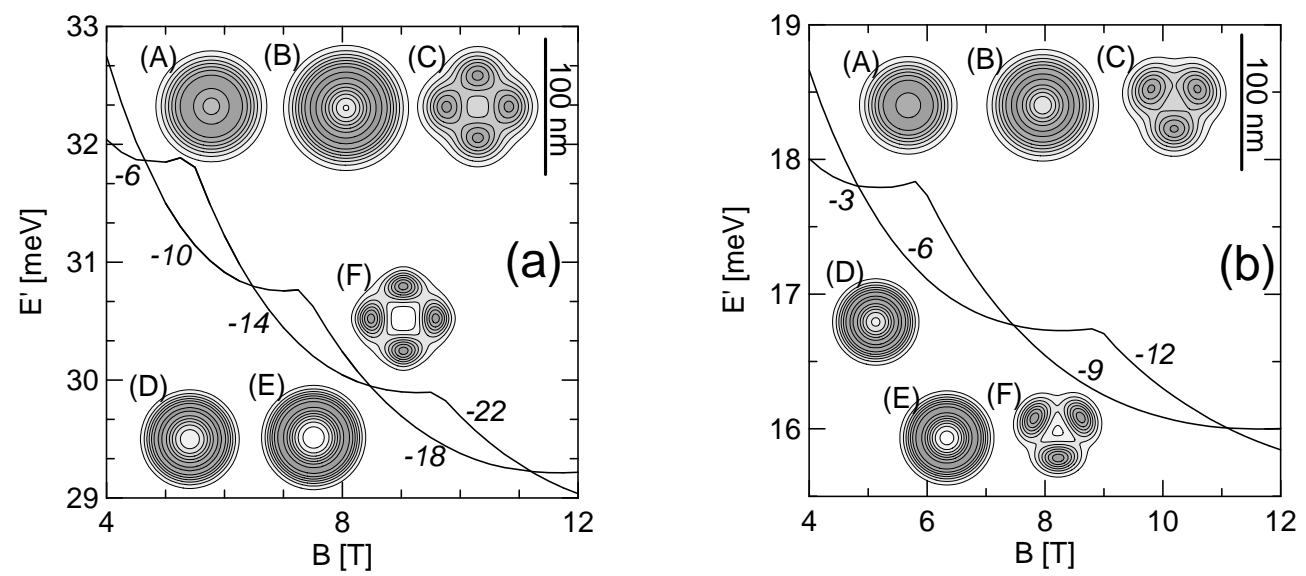

Fig. 1 - (a) Two lowest energy levels of the 4-electron unperturbed circular QD calculated with respect to the lowest Landau level. Numbers denote $L$ - angular momentum in $\hbar$ units. Insets (A), (B) and (C) display the charge densities of the states for $B=4.6 \mathrm{~T}$ corresponding to $L=-6,-10$ and broken symmetry, respectively (the darker the shade of grey the larger the density). (D), (E) and (F) show the charge density of the degenerate states with $L=-18,-22$ and broken symmetry for $B=11.2 \mathrm{~T}$. (b) Same as (a) but for 3 electrons. (A) (B) and (C) plotted for $B=4.2 \mathrm{~T}$ correspond to $L=-3$, -6 and broken symmetry, respectively. (D), (E) and (F) show the charge density of degenerate states with $L=-9,-12$ and broken symmetry for $B=11.2 \mathrm{~T}$.

circular symmetry. In the calculations for the perturbed QDs we used 12 centers placed on an ellipse, the size and its center of gravity were optimized variationally.

Fig. 1 shows the two lowest 4- (a) and 3- (b) electron energy levels calculated with respect to the lowest Landau level $\left(E^{\prime}=E-N \times 0.85[\mathrm{meV} / \mathrm{T}]\right)$ as functions of the magnetic field. $E^{\prime}$ at high field tends to the potential energy of a classical point charge system [15]. At lower magnetic fields the ground state is the MDD with angular momentum $-N(N-1) \hbar / 2$. At larger magnetic fields the angular momentum decreases by $N \hbar[4,5,11]$. The ground-state charge density after the MDD decay has a ring-like shape with a pronounced minimum at the center of the dot. At each ground-state transformation the central local minimum becomes wider and the size of the charge puddle exhibits a stepwise increase. Between the ground state transformations the magnetic field compresses the charge density which shrinks in a continuous fashion [14].

At the angular momentum transformations the ground-state charge density is twofold degenerate. Consequently, each linear combination of the degenerate ground-states $\Phi_{1}$ and $\Phi_{2}$ is also an eigenstate. Consider the following combination $\Phi_{b s}=\left(\Phi_{1}+c \Phi_{2}\right) / \sqrt{2}$, with $|c|^{2}=1$. Since the angular momenta of degenerate ground states differ by $N \hbar$ the angular momentum in state $\Phi_{b s}$ is not defined and $\Phi_{b s}$ possesses a broken-symmetry charge distribution [cf. insets (C) and (F) in fig. 1]. The charge density of the exact broken-symmetry states resembles the approximate mean-field broken-symmetry solutions [4]. The broken-symmetry charge distributions at high field tend [16] to the classical lowest-energy distribution of point charges [10]. Superposition $\Phi_{b s}$ extracts the inner symmetry of the magic angular momenta states into the laboratory frame of reference. The broken-symmetry charge distribution can be oriented at an arbitrary angle depending on the phase of $c$.

Let us now suppose that at a certain distance of the quantum dot plane there is an 
impurity ion located off the symmetry axis of the dot. In vertical quantum dots [12] for which the harmonic approximation of the potential is justified [13], and in which the MDD decay has been observed [12], ionized [13] donor impurities are present at a distance of 20-30 nm from the QD plane. The defect potential perturbs the QD circular symmetry and mixes the angular momentum eigenstates. Level crossings are replaced by avoided crossings. Fig. 2 (a) shows the two lowest-energy levels and the ground state charge density for 4 electrons with a positively charged defect situated at point $x=20, y=0, z=40 \mathrm{~nm}$. The energy gaps in the avoided crossings are very small $\left(\sim 10^{-3} \mathrm{meV}\right)$. At the avoided level crossings (see insets for $B=4.9$ and $6.86 \mathrm{~T}$ ) Wigner crystallization in the laboratory-frame (i.e. WM pinning) can be observed. The positions of the pinned charge density maxima coincides with the position of classical electrons in the lowest energy configurations [cf. lowest inset of fig. 2(a)]. The charge density plots for the magnetic fields outside the avoided level crossings resembles the unperturbed circular densities [cf. fig. 1(a)], although an increased density at the right end of the charge puddle is visible. Since the 'momentary' pinning is a consequence of the existence of the exact broken-symmetry states it appears for an arbitrarily far situated defect.
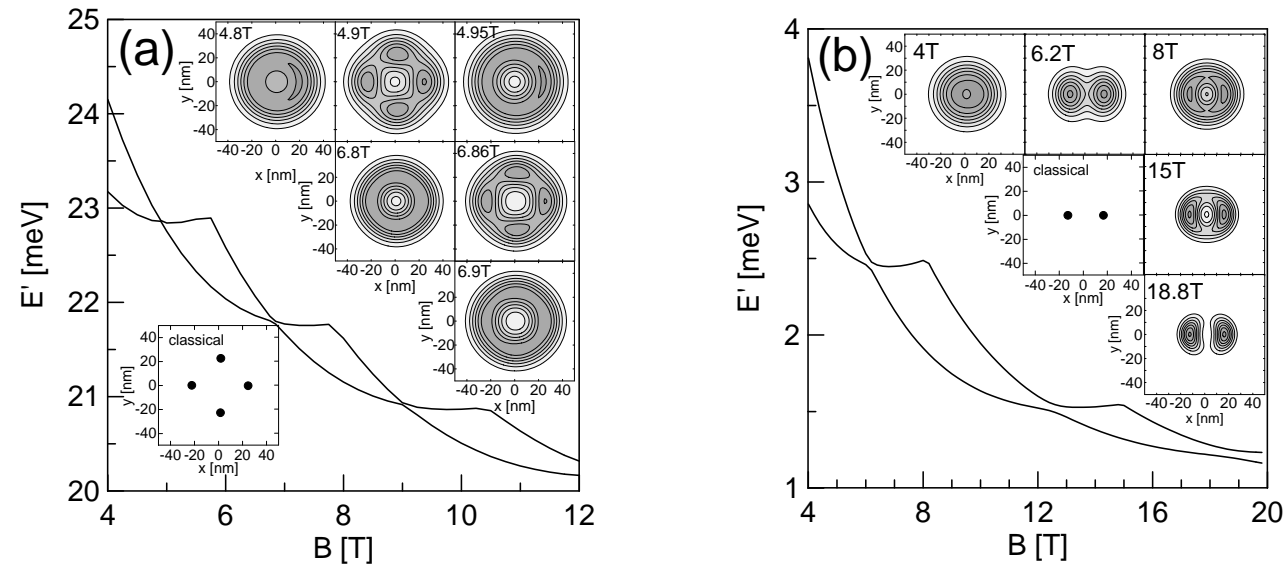

Fig. 2 - (a) Two lowest-energy levels of the 4-electron system in a circular QD perturbed by a potential of a positively charged defect situated at $(20,0,40) \mathrm{nm}$. Insets show the ground state charge densities and the lowest-energy configuration of the classical system. (b) Same as (a) but for 2-electrons.

Fig. 2(b) shows that the effect of the defect on the 2-electron spectrum and the charge density is much stronger [energy gaps are about $5 \times 10^{-2} \mathrm{meV}$ ]. The oscillatory character of the pinning as function of the magnetic field is visible. At avoided level crossings separation of the electron charges is particularly pronounced (see insets for 6.2 and $18.8 \mathrm{~T}$ ). The effect of the negatively charged defect at this rather large distance from the QD is similar, although the molecules become pinned at different angles.

The pinning effect is stronger when the defect is closer to the QD plane. In the rest of the paper we consider a defect located at $(20,0,20) \mathrm{nm}$. Fig. 3 shows the results for 2 electrons. An attractive impurity [fig. 3(a)] enhances the harmonic QD potential which results in a stronger charge localization and as a consequence shifts the anticrossings to higher values of the magnetic field. The energy gap between the lowest levels is larger for repulsive defect [fig. 31 b)]. In both systems an anticrossing related with the MDD breakdown is visible [ $7 \mathrm{~T}$ in (a) and $\sim 5 \mathrm{~T}$ in (b)]. Both systems present smooth non-oscillatory convergence to the 

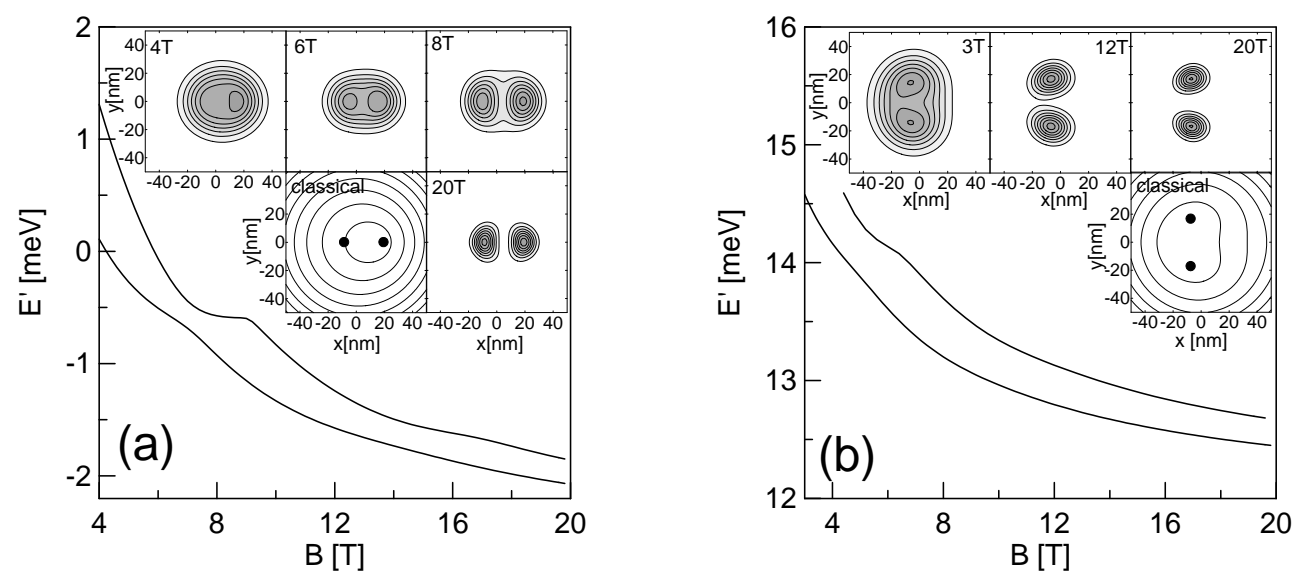

Fig. 3 - Two lowest-energy levels of the 2-electron system in a QD perturbed by (a)-positively and (b)negatively charged impurity situated at $(20,0,20) \mathrm{nm}$. Insets show the ground state charge densities as well as the classical configurations on a background of potential profile (the equipotential lines are spaced by $3 \mathrm{meV})$.

lowest-energy configuration of their classical counterparts.
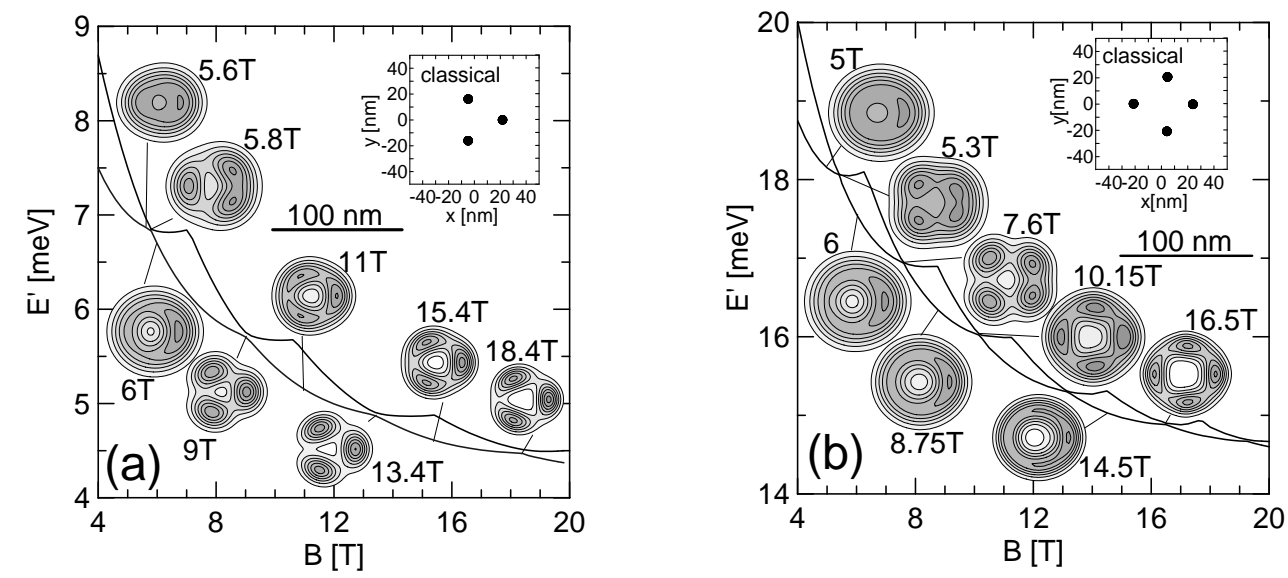

Fig. 4 - (a) Two lowest-energy levels of the 3-electron system in a QD perturbed by a positively charged defect situated at $(20,0,20) \mathrm{nm}$. Insets show the ground state charge densities as well as the classical lowest-energy configuration. (b) Same as (a) but for 4 electrons.

Fig. 4 shows the plots for an attractive impurity with $N=3$ (a) and $N=4$ (b). For both $N=3$ and 4 the energy gaps between the anticrossing levels remain small [around 0.01 meV $(0.04 \mathrm{meV})$ for $N=4(3)$ ] and the pinning of the WMs exhibits anew the oscillatory dependence on the magnetic field. The distribution of charge maxima in the WMs pinned at the MDD breakdown (5.8 $\mathrm{T}$ for $N=3$ and $5.3 \mathrm{~T}$ for $N=4$ ) differs from their classical counterparts. In classical systems a single electron is trapped under the attractive impurity. 
EUROPHYSICS LETTERS

In the WM pinned at the MDD breakdown 2 electrons fit in the local minimum of the potential induced by the defect. At higher fields ( $9 \mathrm{~T}$ for $N=3$ and $10.15 \mathrm{~T}$ for $N=4)$ the pinning fixes the charge maxima near the equilibrium positions of classical electrons. Thus an interesting rotation of the pinned WM is found as function of the magnetic field. The change in the charge distribution in the WMs between the MDD decay and the classical limit is similar to the magnetic-field-induced transformations of the WMs in circular dots for larger $N$ [16]. At high magnetic field the 3 -electron charge density acquires the semi-classical charge distribution even between the anticrossings [cf. plots for 11 and $15.4 \mathrm{~T}$ in fig. 4(a)]. This is not observed for $N=4$ in the studied magnetic field range.
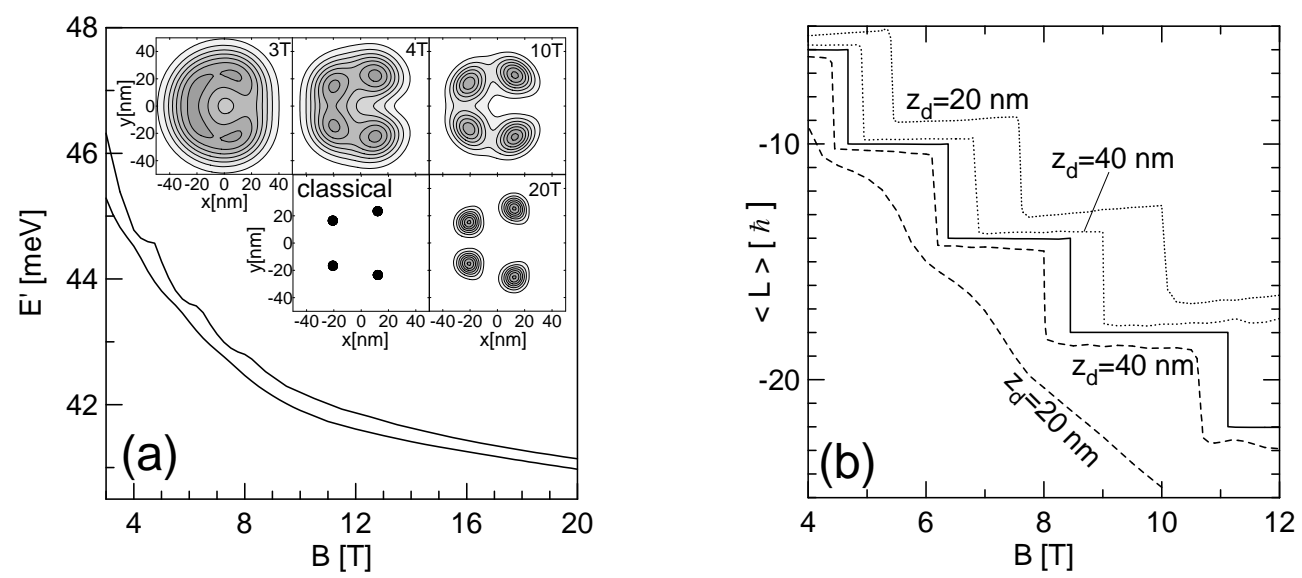

Fig. 5 - (a) Same as Fig. 4 (b) but for a negatively charged defect. (b) Average value of the total angular momentum for 4 electrons without the defect (solid line), in the presence of an attractive (dotted lines) and repulsive (dashed lines) defects at $\left(20 \mathrm{~nm}, 0, z_{d}\right)$

The results for 4 electrons in the presence of a repulsive defect are shown in Fig. 5. The ground state energy is a smooth function of the magnetic field and oscillations appear only in the excited state. A continuous MDD decay appears around $4 \mathrm{~T}$. The charge density tends in a non-oscillatory way to the classical limit of point charges.

The influence of the charged defects on the average value of the total angular momentum for 4 electrons is shown in Fig. 5(b). In the presence of a defect the average values of angular momentum take non-integer values and their dependence on the magnetic field becomes continuous, however much of the stepwise character of a pure QD is conserved for the positive impurity as well as for a distant negative deffect. For the positive (negative) defect the electrons become localized closer (further) from the origin which results in a decrease (increase) of the absolute value of the angular momentum with respect to the unperturbed case. For a negative deffect closer to QD plane the average value is a smoothly decreasing function of the magnetic field. This fast increase of the absolute value of angular momentum is related to the localization of the charge density near the classical equilibrium points [cf. Fig. 5]. Results for 3 electrons for this position of the negative defect are qualitatively the same as for 4 electrons.

Comparing the results for an attractive with those of a repulsive defect [cf. figs 4 and 5 ] shows that the pinning is much more effective in case of a repulsive defect. The attractive defect enhances the confinement potential of the QD, decreases its size and hinders the Wigner crystallization itself. Moreover, it binds one of the electrons in its neighborhood. The potential 
of the bound electron and the defect potential partially cancels and as a consequence the other electrons see a nearly circular potential and the system in the external magnetic field behaves essentially like a $N-1$ electron system. On the other hand, the potential of the repulsive defect is not screened, so it breaks the circular symmetry of the potential felt by each of the electrons in a more pronounced manner.

In conclusion, magnetic-field induced WMs in circular dots are from their very nature susceptible to pinning by the potential of an external charged defect at the angular momentum transitions. Our results can be summarized as follows 1) At large distance between the QD plane and the defect the pinning has a re-entrant character, i.e., it appears only at the energy level anticrossings, which are situated near the angular momentum transition fields of the unperturbed system. 2) For an impurity placed closer to the QD plane, the pinning by the repulsive defect is more effective and leads to a non-oscillatory convergence of the charge density to the classical limit at high field for all $N$. The pinning effect of a positively charged defect is strong only for two electrons. For larger numbers of electrons it is weakened by a partial screening of the defect potential by an electron trapped in the defects neighborhood so that the re-entrant pinning behaviour is conserved. 3) For a positively charged defect close to the QD a magnetic field induced re-orientation of the WM is predicted.

$$
* * *
$$

This paper has been supported by the Polish Ministry of Scientific Research and Information Technology in the framework of the solicited grant PBZ-MIN-008/P03/2003, the Flemish Science Foundation (FWO-Vl), the Belgian Science Policy and the University of Antwerpen (VIS and GOA). One of us (BS) is supported by the Foundation for Polish Science (FNP).

\section{REFERENCES}

[1] Wigner F.P., Phys. Rev., 46 (1934) 1002.

[2] Egger R., Häusler W., Mak C.H. and Grabert H., Phys. Rev. Lett., 82 (1999) 3320.

[3] Müller H.-M. and Koonin S.E., Phys. Rev. B, 54 (1996) 14532.

[4] Reimann S.M. and Manninen M., Rev. Mod. Phys., 74 (2003) 1283.

[5] Maksym P.A., Immamura H., Mallon. G.P. and Aoki H., J. Phys. Condens. Matter, 12 (2000) R299.

[6] Pudalov V.M., D’Orio M., Kravchenko S.V. and Campbell J.W., Phys. Rev. Lett., 70 (1993) 1866.

[7] Vdovin E.E. ,Levin A., Patanè A., Eaves L., Main P.C., Khanin N.Yu., Dubrovkit Yu.V., Henini M. and Hill G., Science, 290 (2000) 122.

[8] Manninen M., Koskinen M., Reimann S.M., and Mottelson B., Eur. Phys. J. D., 16 (2001) 381.

[9] Reusch B. and Egger R., Europhys. Lett, 64 (2003) 84.

[10] Bedanov V.M. and Peeters F.M., Phys. Rev. B, 49 (1994) 2667.

[11] Maksym P.A., Phys. Rev. B, 53 (1996) 10871.

[12] Oosterkamp T.H., Janssen J.W., Kouwenhoven L.P., Austing D.G., Honda T. and TARucha S., Phys. Rev. Lett., 82 (1999) 2931.

[13] Bednarek S., Szafran B. and Adamowski J., Phys. Rev. B, 64 (2001) 195303.

[14] Tavernier M.B., Anisimovas E., Peeters F.M., Szafran B., Adamowski J., and Bednarek S, Phys. Rev. B, 69 (2003) 205305.

[15] Kainz J., Mikhailov S.A., Wensauer A., and Rössler U., Phys. Rev. B, 65 (2002) 115305.

[16] Szafran B., Bednarek S., and Adamowski J., Phys. Rev. B, 67 (2003) 045311.

[17] Yannouleas C. and Landman U., Phys. Rev. B, 68 (2003) 035326. 